\title{
Chaouabtis/oushabtis et leurs fonctions au sein de la religion funéraire des égyptiens anciens
}

\author{
Shabtis / ushabits and their functions in the \\ funerary religion of the ancient Egyptians \\ Shabtis / Ushabtis e suas funções na religião \\ funerária dos egípcios antigos
}

\section{RESUMÉ}

Ce bref article a comme but de présenter le développement de l'usage des chaouabtis/oushebtis en comparaison avec les changements observés dans la religion funéraire des égyptiens anciens, principalement durant le Nouvel Empire. Par le biais d'une révision bibliographique associée à des nouvelles découvertes archéologiques et épigraphiques, ce travail souhaite montrer les diverses interprétations associées à l'usage de cet artefact si courant dans le domaine funéraire.

Mots-clés: Chaouabtis - Oushabtis - Religion funéraire - Équipement funéraire

\begin{abstract}
This brief article aims to present the development of the use of shabtis / ushabtis in comparison with the changes observed in the funerary religion of the ancient Egyptians, especially during the New Kingdom. Through a bibliographical revision associated to the new archaeological and epigraphic discoveries, this paper intends to show the different interpretations connected to the study of this kind of archaeological record so common in the funerary domain.
\end{abstract}

Keywords: Shabtis - Ushabtis - Funerary religion - Funerary equipment

\section{RESUMO}

Este breve artigo tem como objetivo apresentar o desenvolvimento do uso dos shabtis/ushabtis em comparação com as mudanças observadas na religião funerária dos egípcios antigos, principalmente durante o Novo Império. Por meio de uma revisão bibliográfica associada às novas descobertas arqueológicas e epigráficas, este trabalho pretende mostrar as diversas interpretações associadas ao estudo desse artefato tão comum no domínio funerário.

Palavras-Chave: Shabtis - Ushabtis - Religião funerária - Equipamento funerário

* Doutora em Religion et systèmes de pensés du monde antique: égyptologie pela École Pratique des Hautes Études - EPHE. Atua como professora nas Faculdades Metropolitanas Unidas. Pós-Doutoranda em Arqueologia junto ao Museu Nacional (MN), Brasil, junto ao Laboratório de Egiptologia. CV: http://lattes.cnpq. $\underline{\mathrm{br} / 2837896631311697}$ 


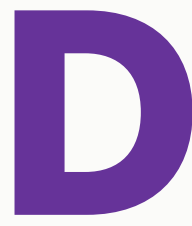

e manière générale on peut observer que les chaouabtis (photo 1) ont eu une diversité d'interprétations fonctionnelles dans leur histoire de deux mille ans d'utilisation et leur vision par les Égyptiens n'est pas restée immuable. Dans un premier temps, ces statuettes se sont manifestées comme un moyen de remplacer les morts physiquement, c'est-à-dire, de dépositoires du Ka, servant pour la manutention de la mémoire, ainsi que de remplaçants pour les travaux agricoles et le maintien de la nourriture des morts dans l'au-delà; car ces figurines qui semblent être apparues avec la disparition des modèles de l'Ancien Empire, travaillent non seulement pour les morts, mais aussi à la place des morts, en étant des doubles du défunt qui labourent, et également des images magiques du propriétaire en soi qui seraient une garantie en cas de destruction du corps.

Photo 1 - Chaouabti de Ramsès VI Amonherkepshef II Nebmaâtrê Meryamon CAIRE CG 48415 JE 96857 SR $4 / 2341 / 0$

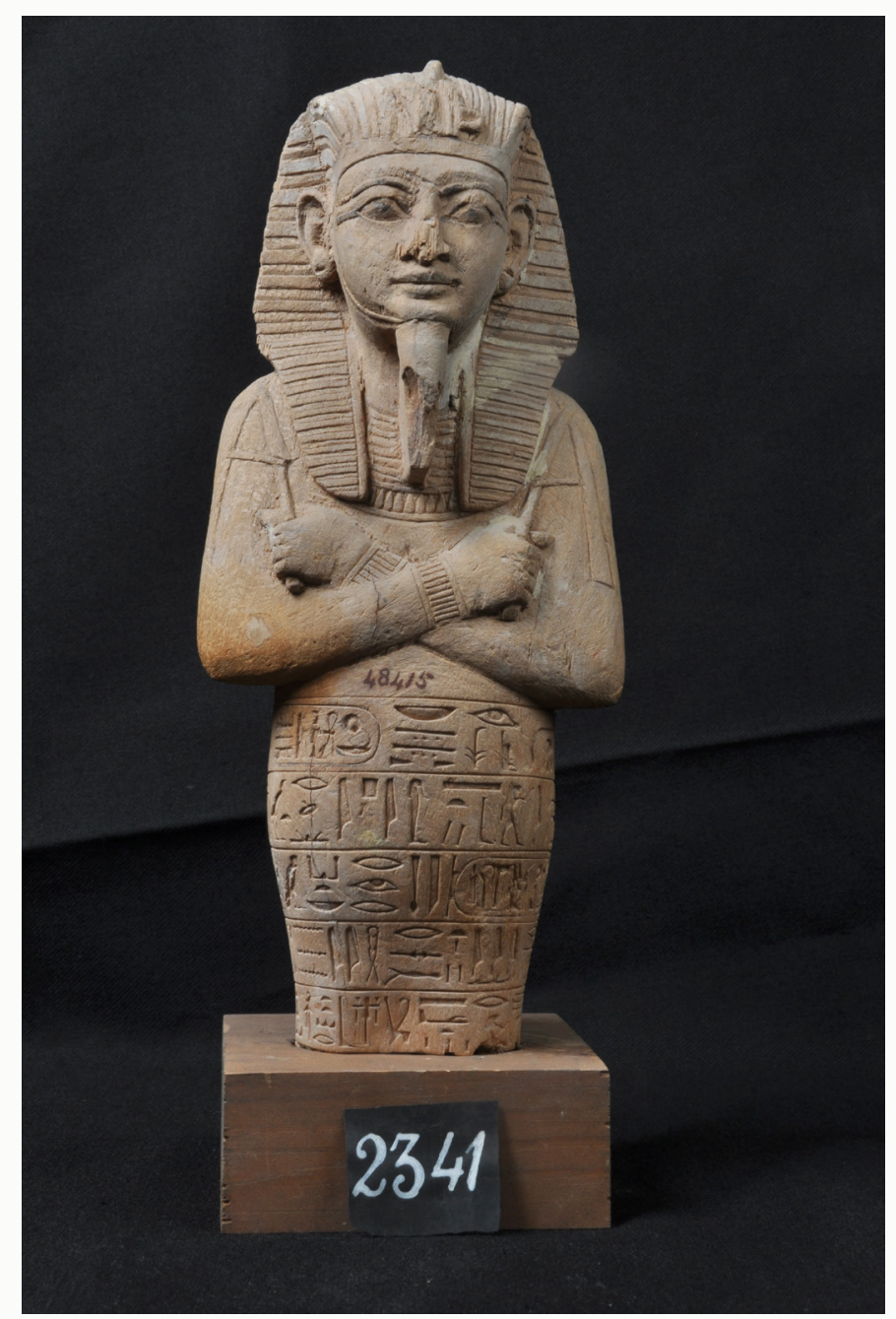

Source: Photo de l'auteur

En comparant les chaouabtis avec, si on peut dire, leurs «prédécesseurs», nous voyons que la différence fondamentale entre les deux groupes, c'est que les modèles de l'Ancien Empire 
travaillent pour le mort, en contrepartie, les chaouabtis le font à la place des morts, car ils ne sont plus représentés en tant que serviteurs en action, mais comme des figurines momiformes. Cependant, tout au long de l'histoire des chaouabtis, ce mode d'action commence à s'altérer, surtout à partir de la fin du Nouvel Empire, ce qui se vérifie par un changement typologique, quantitatif, et même de dénomination - passage de chaouabti à ouchabti, de sorte que des travailleurs qui accomplissaient leurs tâches à la place de leurs propriétaires, en viennent à travailler comme des serviteurs, et non plus comme des remplaçants, de la même manière que les serviteurs de l'Ancien Empire, étaient des ouvriers et non des représentations du mort qui travaille.

\section{Une chronologie.}

Il est difficile d'établir une évolution de la signification des chaouabtis en fonction de l'époque, car plusieurs conceptions sur le caractère de ces figurines semblent avoir coexisté et évolué au cours de ces plus de deux millénaires d'existence. Cependant, on voit que la nature des chaouabtis change peu à peu à la fin du Nouvel Empire.

Avec cela, nous pouvons dire que les serviteurs funéraires ont eu deux phases d'utilisation. La première en tant que doubles/substituts des morts qui agissent comme des travailleurs à la place du mort et la seconde, lorsque les statuettes commencent à être appelées ouchabtis, à la XXle dynastie, en tant que travailleurs dépersonnalisés qui œuvrent comme domestiques pour les morts, étant propriétés du défunt et non le défunt lui-même.

Ce changement de caractère des figurines semble avoir débuté dès la fin de la XVIIIe dynastie, quand les statuettes commencent à prendre des formes diverses et sont placées en plus grandes quantités dans les tombes, atteignant le nombre de 401 figurines à la fin du Nouvel Empire. Ces variations ont pour résultat le fait que les statuettes de la période ramesside, XIXe et XXe dynasties, prennent de nouveaux aspects, pouvant être momiformes ou habillées avec des vêtements des vivants (photo 2), ce qui nous amène à penser que deux groupes distincts commencent à apparaître: les figurines portant les vêtements quotidiens des vivants qui représentent les contremaîtres et les momiformes en tant que travailleurs, ce qui est prouvé par la suite avec l'apparition des surintendants avec leurs fouets et bâtons, qui existeront jusqu'à la XXIIle dynastie. 
Photo 2 - Chaoubti en costume des vivants de Siptah Akhenrê-setepenrê New York MMA 44.4.70
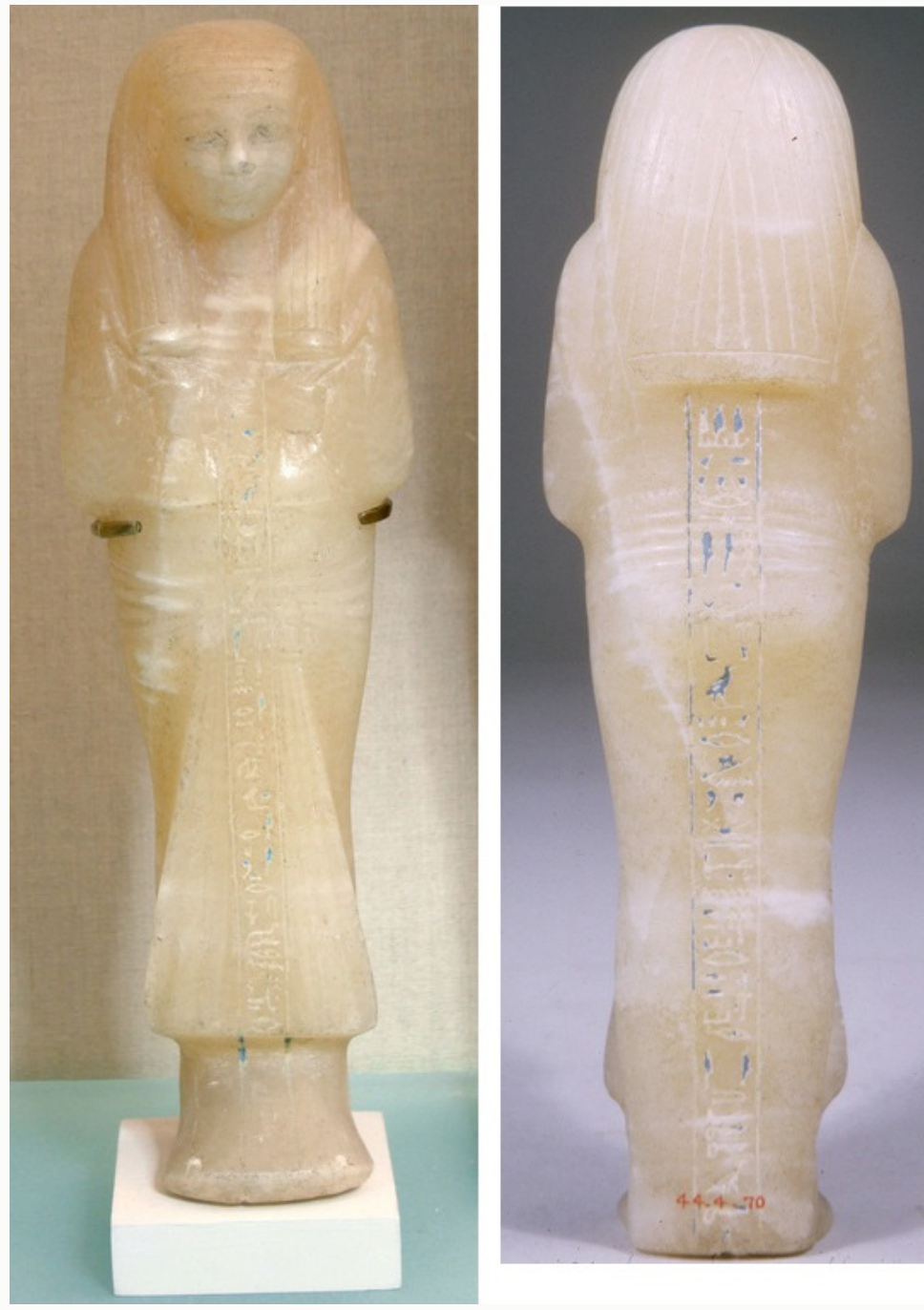

Source: Photo de l'auteur

Dans le monde des vivants un grand groupe d'ouvriers devait certainement travailler sous un certain système organisationnel, qui assurait l'exécution des travaux. Ainsi, il n'est pas étonnant de voir la présence des surintendants dans le monde des vivants et, par la suite, un double de ceux-ci dans le monde des morts, responsable du bon fonctionnement des activités agricoles. ${ }^{1}$

Enfin, à la Période Saïte et à la Basse Époque, lorsque la production des figurines est uniformisée, la séparation entre les deux groupes ne semble plus exister puisque tous sont momiformes, suite à la disparition des surintendants, avec seulement des différences internes au sein d'une troupe du même propriétaire. Nous pouvons évoquer, par exemple, les figurines avec le texte complet du chapitre VI du Livre des Morts, dans sa septième version, et des statuettes plus simples qui ne comptent que le nom et les titres du propriétaire. Par conséquent, nous ne pouvons pas avoir les preuves de l'existence d'une différence de statut entre ces statuettes, c'est-à-dire entre les ouvriers et le surintendant, car on ne voit plus d'instruments pour punir ou

1 Voir les comptes hiératiques de Lahoun, UC 32170 (Miniaci, 2014, p. 267). 
forcer un autre à travailler, les deux étant momiformes et avec les mêmes attributs. De ce fait, de même que nous ne pouvons pas dire qu'il existe une différence de statut entre ces statuettes, nous ne pouvons pas davantage suggérer qu'ils redeviennent des substituts physiques du mort. En effet, même si ces statuettes sont toutes momiformes, elles possèdent les attributs caractéristiques nécessaires pour le travail agricole, houes, sac et pics, et sont déposées en grandes quantités dans chaque sépulture, ce qui est le résultat d'une dépersonnalisation de ces figurines en tant qu'ouvriers (photo 3).
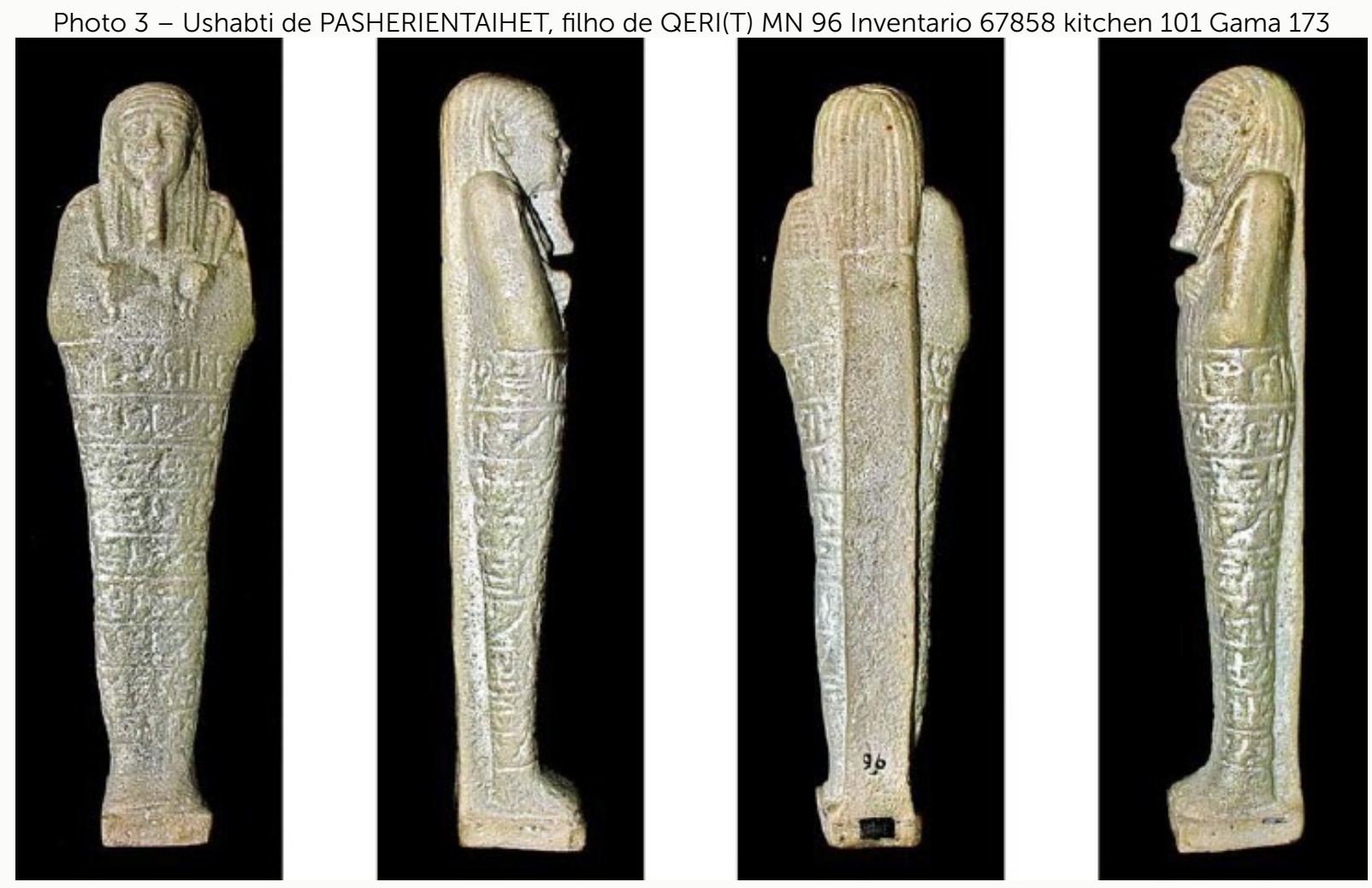

Source: Photo de l'auteur

Par conséquent, on peut dire que le caractère de ces figurines comporte trois phases distinctes, au début du Nouvel Empire, à la fin du Nouvel Empire et à la Basse Epoque. Dans un premier temps, les chaouabtis représentent le mort lui-même et, parce qu'ils sont exactement une copie du "glorifié», ils peuvent être des substituts aux travaux agricoles (pour les rois, ils gardent même les attributs royaux); par la suite, les statuettes ne sont plus le mort en luimême et agissent en tant que serviteurs sous la supervision d'un contremaître, ${ }^{2}$ étant activées pour l'exécution magique d'un travail; enfin, dans la troisième phase, les figurines perdent leur caractère de simples serviteurs, tout en restant des travailleurs, mais, momiformes, désormais plus associées au mort lui-même, mais sans reprendre le caractère originel.

Subséquemment, le Nouvel Empire et le développement des chaouabtis royaux, premiers à présenter une multiplication évidente de leur nombre, correspondent à la période

2 Pour les rois du Nouvel Empire, nous pouvons supposer que les figurines avec regalia sont de contremaîtres et les momiformes des serviteurs, comme cela est observé pour la troupe de Toutânkhamon. 
clé pour observer les changements au sein de la société égyptienne, qui ont mené aux différents moments de l'usage des chaouabtis.

\section{L'usage et signification: des remplaçants?}

Une enquête rapide sur l'iconographie des chaouabtis et sur leurs inscriptions montre ensuite que deux principes de base sont impliqués dans l'interprétation de la signification et des fonctions de ces statuettes:

- Le besoin de nourriture dans l'au-delà pour le propriétaire de chaouabtis, ce qui implique un travail pour les morts;

- L'obligation que la nourriture soit produite par le mort lui-même: autrement dit, la nécessité d'un travail exécuté par les trépassés, ce qui génère la nécessité d'un substitut pour travailler à leur place;

À première vue, l'action des chaouabtis paraît paradoxale, car ils ont la forme d'une momie, mais transportent des outils agricoles, pouvant, parallèlement apparaître en vêtements de vivants. Cependant, ce qui peut sembler contradictoire sous un angle de vue moderne, peut être bien expliqué par la capacité égyptienne de transformer des oppositions en unités complémentaires.

Les chaouabtis incorporent, donc, l'idée des maîtres (les propriétaires de la tombe Osiris - N) et serviteurs, ainsi que le processus de production alimentaire de l'irrigation, à la production finale et à la consommation du produit, ce qui fait que ces petites figurines représentent, de manière globale, la société égyptienne; elles sont les producteurs et les consommateurs, en tant que représentants du défunt, dépositaires du ka et des remplaçants pour le maître sous la forme du propriétaire, ainsi que du travailleur, dans un premier temps, jusqu'à la XXle dynastie, date à laquelle les chaouabtis ne sont pas encore de simples serviteurs (Schneider, 1977, p. 8).

Le rôle du chaouabti comme substitut du maître/mort et serviteur fait partie d'une coutume égyptienne plus générale qui peut être appelée substitution magique. Le remplacement magique était effectué par les Égyptiens dans plusieurs domaines: une personne désignée pour une tâche pouvait être remplacée par une autre, le fils aîné lors du culte funéraire remplacé par le prêtre sa-meref et le prêtre sa-meref pouvait prendre la place de prêtre sem et ainsi de suite.

Dans l'au-delà, le père peut transférer ses obligations envers son fils, qui peut demander à son enfant de quitter la terre pour prendre sa place ou même pour être son représentant sur terre $^{3}$ (Carrier, 2004, p. 76-85; Willems, 2008, p. 193-202). Dans la mythologie, le remplacement peut être une explication à un phénomène, tel que le soleil qui désigne Thot pour prendre sa place, amenant ce dernier à devenir la Lune (Schneider, 1977, p. 69).

Beaucoup de civilisations ont pratiqué et pratiquent la substitution magique. En Égypte aussi, les images remplaceraient le réel et, par des moyens magiques, cette réalité se poursuivrait dans l'éternité. Les images remplacent les personnes, ainsi que les animaux et les

3 Texte des sarcophages, spell 38 et 39. DE BUCK, Adriaan. The Egyptian Coffin Texts 1: Texts of Spells 1-75. Oriental Institute Publications. Chicago, v. 34, 1935. 426p., I 157-I 172 
objets, et puisque le remplacement ne se produit que par l'identification, qui n'est possible que par la puissance magique des mots, le nom de celui qui est remplacé ou un spell associé au remplacement sont essentiels. Ainsi, les chaouabtis semblent avoir les attributs nécessaires pour la substitution magique: un texte associé à des notions magiques et une identification avec le défunt.

Ce remplacement magique est également efficace lors du changement du statut de ces figurines, autrement dit, quand ils deviennent ouchabtis et agissent en tant que serviteurs et producteurs d'aliments, car les éléments de base magico-religieux nécessaires à la substitution magique sont maintenus. Toutefois, même si ce sont des serviteurs, ils peuvent encore être considérés comme affiliés au défunt, parce qu'ils n'ont pas abandonné leur caractère momiforme, étant des représentations des esprits «glorifiés», akhou, possédant des outils agricoles.

\section{Les chaouabtis/ oushabtis et le Akh.}

Le caractère momiforme des chaouabtis les associe aussi aux akhw. Mais qu'est-ce exactement que l'akh?

Akh serait l'esprit lumineux, le mort transformé ou transfiguré après les rites funéraires, un défunt devenu efficace (Friedman, 1984-1985, p. 40 et 46). Après avoir mené une vie selon la maât, avoir subi la momification, les rites funéraires, avoir passé toutes les épreuves et obstacles du monde souterrain et avoir bénéficié des formules magiques sahu - formules rituelles «pour faire que quelqu'un devienne un akh» (Friedman, 2001, p. 47) - et ensuite être enterré, le défunt peut finalement être glorifié ou transfiguré en akh, pouvant être considéré comme une étoile (Friedman, 1984-1985, p. 45-46). Sous cette forme, il gagne des attributions (Englund, 1978, p. 17-18) qui dépassent les limites entre le monde des vivants et des morts, devenant un intermédiaire entre les sphères du vivant et du divin en tant qu'être efficace (Friedman, 1982, p. 145-148; 2001, p. 47-48).

Quand un mort a réussi à devenir un akh, après avoir été justifié et transfiguré, il se transforme en une entité qui participe à la sphère divine (Smith, 2006, p. 334) ${ }^{4}$ ainsi qu'à la sphère humaine et au monde des vivants. Les akhou gardent leurs tombes, ils interagissent avec les vivants en aidant avec des capacités qu'ils n'avaient pas de leur vivant, en agissant comme médiateurs entre les vivants et les dieux ou entre les vivants et d'autres akhou, comme on peut l'observer sur les lettres aux morts.

Même avec ce statut spécial, les akhou ont aussi besoin des vivants, car ce sont ces derniers qui accomplissent les rituels et font les offrandes aux ancêtres décédés (Friedman, 1981, p. 137-138). Les besoins des akhou sont attestés par la cérémonie appelée «l'alimentation de l'akh» avec le mort glorifié représenté devant les offrandes alimentaires dans les tombes des IVe et Ve dynasties. Cette cérémonie a été postérieurement incorporée au rituel d'ouverture de

4 Nous pouvons mettre en relation le fait de devenir un akh avec l'intégration de la sphère osirienne par le biais de l'épithète Osiris un tel. Voir 3.7.12. 
la bouche. De cette façon, les esprits glorifiés et les vivants ont une relation de co-dépendance, comme on peut l'observer par la relation d'un père décédé avec son fils. Le fils doit assurer les rituels et les offrandes pour son père, ce qui lui permet d'accomplir des actions qui l'autorisent à devenir, après sa mort un akh. Sur le plan des vivants, le fils doit prendre la position et l'autorité de son père et le père décédé protège et soutien de l'au-delà, le fils vivant. Ce type d'efficacité mutuelle entre père et fils trouve son modèle mythique dans la relation entre Osiris et Horus (Friedman, 1984-1985, p. 43-44).

Cette relation d'efficacité et co-dépendance est aussi visible entre les rois et les dieux. De son vivant, le roi doit être efficace, akh, pour les dieux, en construisant des temples ou monuments, en agissant comme le fils auprès de son père qui est dans l'au-delà (Friedman, 1984-1985, p. 40). Cette efficacité du roi envers le dieu est évidente pour le roi Akhenaton, où le mot akh est mis en évidence dès son prénom: le roi se manifeste ainsi dans une relation de réciprocité avec le dieu, son père divin; il est akh - efficace - et il accomplit des akhwt choses effectives - pour son père qui lui rend la pareille à son tour. Par exemple, le roi lui fait construire des monuments et le dieu, en retour lui donne vie et des millions de heb-sed. Cette action efficace doit exister aussi de la part du roi envers ses prédécesseurs et sujets (Friedman, 1982, p. 146). Le roi mort est aussi un akh, dans le sens de glorifié, mais il garde toujours un statut privilégié «à la tête des akhou» ${ }^{5}$ ou comme le premier des akhou, le «akh akhou», de la même façon qu'il l'était en vie.

Nous pouvons de cette manière percevoir deux notions associées au mot akh. L'une est liée au domaine des morts, associée à un état du mort transfiguré, un mort qui est efficace et a bénéficié de toutes les offrandes, rituels et connaît les spells pour l'au-delà. L'autre notion est davantage liée aux vivants avec l'idée d'efficacité, en accomplissant des actions et en remplissant des tâches pour quelqu'un, comme un défunt qui agit pour la protection du vivant et vice-versa (Friedman, 1985, p. 96-97), les rois, les seigneurs et tous les vivants sont akh auprès des dieux et les uns envers les autres.

\section{L'union de deux destinées post-mortem}

Nous pouvons, après cette brève explication placer les chaouabtis en tant que représentations associées au akh, ou même comme une représentation du akh, car ses figurines agissent au nom du mort et à sa place, étant efficaces pour le défunt. D'un point de vue morphologique, ces figurines sont majoritairement représentées sous forme de momie, un mort qui a retrouvé ses capacités grâce aux rituels. Le chaouabti peut être, ainsi, akh pour le défunt.

C'est par l'association entre Osiris et Rê en tant qu'Akh que l'on peut voir une des facettes de l'union de ces deux dieux dans le domaine funéraire. Rê représente, entre autres, le dynamisme de la création, mais même dans ce mécanisme dynamique solaire, il y a, par

5 Textes des Pyramides 833, 858, 869, 899, 903, 1724, 1899, 1913-1914, 2096 et 2103. 
l'occultation de Rê, une association à la mort. Osiris, lui, représente le côté statique de la création, qui est figé, paralysé, au moins momentanément, par la mort, mais qui sort de l'inertie ; le grain qui monte et qui renaît après avoir été enterré, en tant que vie qui a encore un potentiel d'évolution et de dynamisme

On aperçoit, alors, une intention claire de la part des Égyptiens de combiner deux éternités ou deux temps. Une éternité conçue comme un état final devenant immuable, osirien et linéaire, le djet. L'autre, une éternité conçue comme un flux infini du temps, céleste et cyclique (Assmann, 2003, p. 111-112), neheh. La première est associée au corps, placé dans le caveau et pérennisé par les rituels funéraires. La deuxième est liée à l'âme-ba, qui se manifeste dans le domaine terrestre (Servajean, 2007, p. 26-27). L'une est le temps par essence mesurable et possédant un début et une fin, auquel tous les êtres d'ici-bas sont soumis et l'autre, djet, l'éternel du monde imperceptible de l'au-delà (Servajean, 2007, p. 36).

Si nous nous considérons l'analyse de F. Servajean (2007, p. 19-20) nous voyons aussi ces deux expressions temporelles associées au temps de la terre, à une durée sociale, à une éternité à l'échelle du temps humain et au ba - neheh - et à une durée divine, éternité qui dépasse la durée de vie terrestre, le corps pérennisé - djet -; ce qui renvoie à l'utilisation des chaouabtis en tant que substituts du mort dans une sphère qui associe toutes les durées, y compris celles du défunt, en tant que remplaçant du défunt, maître et serviteur en même temps. Nous pouvons de cette façon associer le maître, le défunt lui-même, celui à qui appartiennent les serviteurs funéraires, dont le corps est pérennisé et a subi les rituels funéraires à la manifestation éternelle djet. Par la suite, nous pouvons mettre en relation le serviteur, cette manifestation annexe et attachée au défunt qui est «utilisable» comme un double magique, au temps neheh. Ainsi, on associe deux manifestations telles que le corps ritualisé sous sa forme osiriaque et le domaine terrestre et temporel humain avec les outils agricoles et les attributs des vivants.

Cette logique d'association des temps et éternités terrestres et divines, nous permet aussi de comprendre la nécessité des chaouabtis de la part de rois qui n'ont jamais exécuté de travaux agricoles de leur vivant. Ces êtres se placent par principe dans les deux durées, neheh et djet, en tant que personnes soumises à neheh et par leur fonction à djet. Le roi a une durée neheh de son vivant et en a une autre dans l'au-delà (djet) la même que la fonction royale. Même si les souverains emportent les attributs et insignes du pouvoir de leur vivant dans l'audelà, djet, ils sont obligés de réaliser des actions pour les dieux, telles les corvées agricoles, car ils sont, en même temps des akh auprès des dieux, et doivent être efficaces vis-à-vis des dieux après la mort comme ils l'ont été en vie.

La contradiction apparente au regard des chaouabtis, c'est la multiplicité des fonctions qui leurs sont associées; ce sont des serviteurs à la place du mort, des doubles du mort qui travaillent et des travailleurs pour le défunt, momiformes avec des outils agricoles. Toutes ces fonctions ne sont pas incompatibles, c'est une façon de combiner des points de vue, par le biais du remplacement magico-religieux.

La caractéristique la plus permanente et traditionnelle des chaouabtis est leur caractère momiforme, représentation typiquement osirienne, alors que leur matériau est pour la plupart en faïence égyptienne. Sont également attestés des matériaux tels que le bois ou l'argile décorés 
ou recouverts des couleurs associées à la renaissance, à la fois osirienne et solaire. D'un côté le noir et le vert attribués à Osiris, au limon fertile du Nil et à la réactivation des propriétés des sols fertiles et de l'autre la brillance de la faïence liée à une luminosité solaire.

Ces couleurs peuvent aussi être associées à l'utilisation des vocables sehedj Wsir, «illuminé» Osiris, en d'autres mots, celui qui est auprès d'Osiris et est «illuminé», qui est auprès du dieu chthonien et du dieu céleste, une image momiforme qui brille comme la lumière du soleil, un mort «justifié» et "glorifié», un akh, qui vit éternellement et pour toujours, dans les deux domaines de l'au-delà.

En tenant compte des textes du Nouvel Empire sur le monde inférieur, on peut aussi voir que l'union de la momie, le corps d'Osiris, au soleil, surtout dans sa forme criocéphale, lui permet de reprendre sa puissance d'illumination, revenant à la vie tous les matins. Cette union entre le ba et le cadavre du soleil, qui se produit dans le monde souterrain, est réitérée sur les chaouabtis par l'union de la lumière avec le caractère osirien, de la forme avec le matériau.

De plus, sur le papyrus Bodmer 103, de la XXle dynastie, l'un des rares où l'on trouve le chapitre VI du Livre des Morts nous lisons le texte suivant, où l'interpellation s'adresse à deux aspects successifs de l'astre solaire: le lion Routy y est une désignation de Rê-Horakhty ou même d'Atoum et Khépri, et montre déjà une association entre un destin osirien et des caractères solaires.

Formule pour permettre que les répondants exécutent les travaux dans la tombe pour l'Osiris N. Réciter mes paroles "Ô Khépri, ô lion Routy venez à nous permettre que les répondants exécutent pour nous les travaux dans la nécropole, quotidiennement, sans jamais disparaître» (Valloggia, 1991, p. 134-135).

En ce qui concerne le texte, le fait que le nom du défunt soit inscrit sur les statuettes garanti l'efficacité de l'objet, en perpétuant sa mémoire et préservant la vie dans l'au-delà. Le ka doit être maintenu en vie grâce au ravitaillement produit par ces travailleurs, qui exécutent des tâches spécifiées dans le texte des chaouabtis, et à la nourriture produite pour un au-delà osirien qui reproduit l'Égypte des vivants, composé de champs, par lesquels le soleil passe avec son bateau durant la nuit, apportant vie et fertilité et en rejoignant son corps pour refaire surface le lendemain matin.

Finalement, nous pouvons dire que ces petites figurines agissent comme des serviteurs, comme substituts physiques du mort et combinent ces deux fonctions en même temps. Elles représentent aussi une sorte de synthèse des concepts funéraires, qui varient d'une époque à une autre au cours de leur longue période d'utilisation. Leur existence permet au mort de devenir un akh et ces figurines sont aussi des akh auprès du défunt, car elles sont efficaces pour celui-ci et œuvrent pour que tout se déroule de la meilleure manière possible pour leur maîtres/doubles. 


\section{Fin de l'usage des chaouabtis.}

Après l'invasion des Perses, la conquête d'Alexandre n'apporte pas de changements immédiats radicaux aux coutumes funéraires égyptiennes. Toutefois avec la domination lagide on assiste à une accélération du processus de changement des coutumes funéraires, à travers une nouvelle façon de regarder la mort (Schneider, 1977, p. 346-347). Ainsi, selon J. F. et L. Aubert, les figurines ont lentement commencé à tomber en désuétude dans les grands centres (Aubert, 1974, p. 210).

Ce changement dans les coutumes funéraires, qui se traduit par la fin de l'usage des chaouabtis peut déjà être observé à la fin de la Basse Époque à travers la «spiritualisation» de la mort. Désormais, le destin osirien est vu comme un avenir sombre et solitaire où le défunt était constamment affamé et assoiffé, où il devait travailler. Commence alors à se manifester une renaissance d'anciens concepts cosmologiques de l'au-delà, selon lesquels les morts prennent la forme d'une étoile ou s'associent au soleil. La reprise de ces concepts éloigne le défunt de l'au-delà osirien, l'approchant d'une destinée céleste. Ces caractéristiques cosmiques deviennent très courantes dans les textes funéraires et dans l'iconographie funéraire de la période ptolémaïque. Dans le concept traditionnel d'au-delà égyptien, le mort souhaite être identifié avec le dieu Osiris ou à son entourage, devenant un «Osiris N» ou «Osiris n N», et être identifié avec le pouvoir suprême de la vie, Rê-Horakhty. Avec ces identifications mythologiques le mort devient comme un dieu, et obtient le pouvoir magique de surmonter les difficultés du passage à l'autre monde. Toutefois, même en étant comme un dieu, le mort continue d'être un sujet vis-à-vis des seigneurs de l'au-delà. Avec les nouveaux concepts de la nature du mort, qui prennent le dessus à la période ptolémaïque, le défunt s'imprègne de la nature des dieux: il est Osiris et il est Rê en soi, et non plus comme les dieux, il devient un dieu. Avec cette union mystique, qui se passe dans l'au-delà, Osiris et Rê sont des aspects de la déification du défunt. L'individualité terrestre du mort ne change pas, il continue d'être le même, mais sous une nouvelle forme osirienne. Il n'est plus «l'Osiris N» (Wsir N) mais «l'Osiris de N» et la contrepartie féminine est «Hathor N» (Kákosy, 1971, p. 99).

Dans les représentations funéraires le nouvel Osiris mort apparaît pour la première fois à la période ptolémaïque avec des vignettes sur des cartonnages qui montrent le mort portant la couronne atef avec le disque solaire sur sa tête (Kákosy, 1971, p. 98-100). Sur les cartonnages de la période romaine, le mort est représenté comme Osiris avec des emblèmes osiriens dans ses mains (Grimm, 1974, pl. 116,1 et 117,3).

Tous ces changements religieux expliqueraient la disparition des chaouabtis; disparition due à l'affaiblissement de l'au-delà osirien, et, par conséquent des champs osiriens, lieu de travail où le mort était censé travailler le sol et cultiver ses portions de terre, lieu d'action des chaouabtis, ce qui commence à rendre ces objets inutiles (Schneider, 1977, p. 346-347).

Toutefois, pendant un certain temps, ces figurines continuent d'être produites, mais plutôt comme un anachronisme religieux, car leur fonction n'est plus d'actualité, en étant d'une facture plus simple, en terre cuite ou en faïence égyptienne, souvent anépigraphes et avec des formes schématiques. 
Pour J. F. et L. Aubert (Aubert et Aubert, 1974, p. 210), l'usage des serviteurs funéraires se serait éteint au début de l'ère chrétienne, mais sans datation précise, tandis que H. D. Schneider affirme que le dernier chaouabti avec une datation sûre serait la statuette de Pa-shery-en-Ptah, prêtre à Memphis sous le règne de Cléopâtre VII (Schneider, 1977, p. 346-347). Cependant, les chaouabtis de la période ptolémaïque n'ont pas été soumis à une étude approfondie, étant très souvent classés comme tardifs, sans recherche plus détaillée, surtout en ce qui concerne l'épigraphie; ils sont relégués dans les réserves techniques des musées et dans les collections privées et une étude sur ces chaouabtis pourrait compléter nos connaissances sur ces artefacts funéraires.

À l'époque grecque, surtout au dernier siècle avant notre ère, plusieurs statuettes ont été faites en style gréco-égyptien, mais une seule peut être considérée avec certitude comme étant un chaouabti, les autres seraient plutôt des représentations osiriennes du mort ou aussi ce qu'on peut appeler des «pseudo-chaouabtis». Pour que ce soit plus clair nous citons en dessous deux exemples de statuettes gréco-égyptiennes.

La première est une statuette en faïence de $18 \mathrm{~cm}$, momiforme sur une base rectangulaire avec un pilier dorsal. Les bras sont croisés sur la poitrine, le droit sur le gauche et dans la main gauche on aperçoit des lignes suggérant les fibres d'une corbeille qui se prolonge jusqu'au dos passant par l'épaule droite. La tête du personnage n'est ni grecque ni égyptienne, mais plutôt chypriote: un visage large avec des grands yeux ouverts, un sourire, un nez pointu, une barbe courte séparée en deux touffes et une chevelure encadrant le front et retombant sur le cou en rouleau à la mode hellénistique. Nous avons, ainsi, un corps égyptien avec la tête d'un étranger (Maspero, 1902, p. 186-187) ${ }^{6}$. Malheureusement, cette figurine ne porte pas d'inscription, mais la qualité de la faïence et du modelage ainsi que le corps allongé sont caractéristiques des chaouabtis de la XXXe dynastie et de la période ptolémaïque. Des parallèles pour la tête peuvent être trouvés parmi la sculpture chypriote entre le Ve et le Ille siècle (Schneider, 1977, p. 348). G. Maspero avait interprété cette statuette comme une représentation du mort en tant que Sérapis (Maspero, 1902, p. 187). Cependant, à notre avis, cette figurine serait, plutôt, un chaouabti d'un étranger, d'un Chypriote, qui, en suivant la tradition égyptienne, a réalisé un corps osirien, momiforme, avec une possible corbeille tenue par la main gauche. Cet exemplaire de chaouabti grec a été trouvé à Saqqarah sous une accumulation de débris sous la pyramide d'Ounas, une zone remplie de galeries et puits, qui, à la période ptolémaïque, a servi comme lieu de sépulture (Barsanti, 1902, p. 182-184). Ainsi, un objet chypriote dans ce contexte n'est pas surprenant, car, autour de la ville de Memphis, des quartiers étaient réservés aux étrangers. Le propriétaire du dit chaouabti a pu avoir appartenu à cette communauté «helléno-memphite» qui continue la coutume funéraire égyptienne, sans perdre, dans l'au-delà, sa propre identité.

La deuxième statuette citée, considérée comme un «pseudo-chaouabti», est inscrite au nom de Soter, le marin - BM 30769; elle est en faïence noire avec des détails en faïence verte en relief ${ }^{7}$. Avec une tête ronde et une barbe hellénistique, sur la partie dorsale on trouve le nom et le titre, et sur une colonne frontale des pseudo-hiéroglyphes. La statuette est percée

6 Photo indisponible.

Photo non publiée. 
au niveau du cou, ce qui peut signifier qu'elle a été utilisée comme amulette. Par conséquent, la figurine doit être prise comme un ex-voto ou une amulette offerte à un dieu. Cette statuette est très probablement datable de la période romaine (Schneider, 1977, p. 349).

Finalement, on signalera aussi un autre genre de figurines des époques ptolémaïque et romaine, mais qui ne peuvent pas être considérées comme des chaouabtis. Ces statuettes utilisées comme fournisseurs d'aliments ne sont pas des remplaçants du mort et composent une catégorie différente d'objets archéologiques.

Ces statuettes en terre cuite ont été trouvées dans le cimetière de Kôm esh-Shoukafa près d'Alexandrie (Schreiber, 1908, p. 228, 231 et suiv.). Les propriétaires des tombes sont accompagnés par des nombreuses statuettes polychromes de serviteurs, la majorité accroupie et avec les mains vides, mais, quelquefois avec des corbeilles ou boîtes remplies de fruits. Ces statuettes étaient rangées dans des pots qui pourraient aussi contenir des aliments. Ces récipients ont été trouvés près du mort. Ces statuettes ont aussi été retrouvées dans des niches de la chambre funéraire, quelquefois accompagnées de vases avec de la nourriture. Les propriétaires des tombes avec ce genre de figurines étaient grecs ou romains, mais les artefacts semblent avoir été fabriqués par des Égyptiens. Ces serviteurs sont tous en style grec et ne représentent pas des remplaçants pour les travaux dans l'au-delà, mais simplement des travailleurs qui peuvent être comparés aux statuettes des serviteurs de l'Ancien Empire. L'influence égyptienne est, ainsi, possible, mais ne peut pas être prouvée, car l'idée de serviteurs de ce genre peut être originaire de la Grèce ; en effet dans des tombes de Béotie entre les Vle et Ve siècles, on trouve des statuettes similaires, c'est-à-dire de femmes avec des fruits et des boulangers (Köster, 1926, p. 33, pl. 9/11).

Nous savons, cependant, que pendant la période romaine, les chaouabtis deviennent des objets exotiques qui ont été emportés comme souvenirs par les visiteurs de passage en Égypte, ce qui explique que ce type d'objets ait été retrouvé dans diverses régions de l'empire romain, mais sans avoir de fonction funéraire associée au défunt ou au travail dans l'au-delà. Pouvant être des objets anciens remis en circulation ou même des objets fabriqués à l'époque romaine, reproduisant des modèles anciens pour satisfaire un goût égyptianisant des Romains.

Finalement, de la même manière que les figurines funéraires n'ont pas une date précise d'apparition, on ne trouve pas une date pour l'abandon de leur production; en effet, tous ces changements ne se font pas d'un seul coup et suivent un long processus parfois difficilement compréhensible; ce qu'on peut nonobstant constater c'est que leur fin est probablement due à des changements religieux et culturels tout comme leur apparition.

\section{Références}

ASSMANN, Jan. La notion d'éternité dans l'Égypte ancienne. Dans: PIRENNE-DELFORGE,Vinciane et TUNCA, Önhan (Éd.). Représentations du temps dans les religionsi. Liège: Bibliothèque de la Faculté de Philosophie et Lettres de l'Université de Liège, p. 111-122, 2003.

AUBERT, Jacques François et AUBERT, Liliane. Statuettes égyptiennes: chaouabtis, ouchebtis. Paris: Librairie d'Amérique et d'Orient, 1974. 341p. 
BARSANTI, Alexandre. Fouilles autour de la pyramide d'Ounas. Annales du Service des Antiquités de l'Égypte. Le Cairo, n. 3, p. 182-184, 1902.

CARRIER, Claude. Textes des sarcophages du Moyen Empire égyptien. Trois volumes. Paris : Éditions du Rocher, 2004.

ENGLUND, Gertie. Akh: Une notion religieuse dans l'Égypte pharaonique. BOREAS. Uppsala, v. 11, 1978. 227p.

FRIEDMAN, Florence Dunn. Review of G. Englund: Akh: Une notion religieuse dans l'Égypte Pharaonique. Journal of the American Research Center in Egypt. Cairo, v. 19, p. 145-148, 1982.

FRIEDMAN, Florence Dunn The Root Meaning of Ax: Effectiveness or Luminosity. Serapis: The American Journal of Egyptology. Chicago, n. 8, p. 39-46, 1984-1985.

FRIEDMAN, Florence Dunn. On the meaning of some anthropoid busts from Deir le-Medina. Journal of Egyptian Archaeology. London, v. 71, p. 82-97, 1985.

FRIEDMAN, Florence Dunn. Akh. In: REDFORD, Donald. (Ed.). The Oxford Encyclopedia of Ancient Egypt. Oxford: Oxford University Press, p. 47-48, 2001.

GRIMM, Günter. Die römischen Mumienmasken aus Ägypten. Wiesbaden: Steiner, 1974. 199p.

KÁKOSY, László. Selige und Verdammte in der spätägyptischen Religion. Zeitschrift für Ägyptische Sprache und Altertumskunde. Leipzig, v. 97, p. 95-106, 1971.

KÖSTER, August. Die griechischen Terrakotten. Berlin: H. Schoetz, 1926. 89p.

MASPERO, Gaston. Note sur les objets recueillis sous la pyramide d'Ounas. Annales du Service des Antiquités de l'Égypte. Le Cairo, n. 3, p. 185-190, 1902.

MINIACl, Gianluca. The case of the Third Intermediate Period 'shabti-maker (?) of the Amun Domain' Diamun/Padiamun and the changes in conception of shabtis statuettes. Journal of Egyptian Archaeology. London, v. 100, p. 253-282, 2014.

SCHNEIDER, Hans D. Shabtis: an Introduction to the History of Ancient Egyptian Funerary Statuettes with a Catalogue of the Collection of Shabtis in the National Museum of Antiquities at Leiden. Leyde: Rijksmuseum van Oudheden, 1977. 835p.

SCHREIBER, Theodor. Die Nekropole von Kôm-esch-Schukâfa: Expedition Ernst Sieglin in Alexandria. Tome $1^{\text {er. }}$ Leipzig: Giesecke \& Devrient, 1908. 408p.

SERVAJEAN, Frédéric. Djet et Neheh: une histoire du temps égyptien. Orientalia Monspeliensia. Leyden, v. 18, 2007.

SMITH, Mark. Osiris NN or Osiris of NN? Dans: BACKES, Burkhard; MUNRO, Irmtraud et STÖHR Simone. (Éd.) Totenbuch-Forschungen: Gesammelte Beiträge des 2. Internationalen Totenbuch-Symposiums Bonn, 25, September 2005, Wiesbaden, p. 325-337, 2006.

VALLOGGIA, Michel. Le papyrus Bodmer 103: un abrégé du Livre des Morts de la Troisième Période Intermédiaire. Cahier de Recherches de l'Institut de Papyrologie et d'Egyptologie de Lillie. Lilie, n. 13, p. 129-136, 1991. 
WILLEMS, Harco. Les textes des sarcophages et la démocratie: éléments d'une histoire culturelle du Moyen Empire. Paris: Éditions Cybèle, 2008. 284p.

Enviado em: 12 de novembro de 2018

Aprovado em: 10 de dezembro de 2018 